To be published in Optics Letters:

Title: $\quad$ Nonlinear Photoacoutic Response of Suspensions of Laser-Synthesized Plasmonic Titanium Nitride Nanoparticles

Authors: Anderson S. Gomes,Andrei Kabashin,Melissa Maldonado Cantillo,avishek das,Sergey Klimentov,Anton Popov

Accepted: 31 October 20

Posted 10 November 20

DOI: $\quad$ https://doi.org/10.1364/OL.404304

(C) 2020 Optical Society of America 


\title{
Nonlinear Photoacoustic Response of Suspensions of Laser-Synthesized Plasmonic Titanium Nitride Nanoparticles
}

\author{
Melissa E. Maldonado, ${ }^{1}$ Avishek Das, ${ }^{1}$ Anderson S. L. Gomes, ${ }^{1,3, *}$ \\ Anton A. Popov, ${ }^{2}$ Sergey M. Klimentov, ${ }^{2}$ Andrei V. Kabashin ${ }^{2,3}$ \\ ${ }^{1}$ Departamento de Física, Universidade Federal de Pernambuco, Recife-PE 50670-901, Brazil \\ ${ }^{2}$ MEPhl, Institute of Engineering Physics for Biomedicine (PhysBio), Bio-nanophotonics Laboratory, 31 Kashirskoe sh., 115409, Moscow, Russia \\ ${ }^{3}$ Aix Marseille Université, CNRS, LP3, Campus de Luminy, Case 917, 13288, Marseille, France \\ ${ }^{*}$ Corresponding author: anderson.lgomes@ufpe.br
}

Received XX Month XXXX; revisedXX Month, XXXX; accepted XX MonthXXXX; posted XX Month XXXX (Doc. ID XXXXX); published XX Month XXXX

\begin{abstract}
Nonlinear photoacoustic response from solutions of $\mathbf{4 0}$ nm plasmonic titanium nitride (TiN) nanoparticles (NPs) synthesized by laser ablation in liquid environment (acetone) is reported. Using photoacoustic Z-scan with 5 ns pumping pulses, values of effective nonlinear absorption coefficients $\beta_{\mathrm{PA}, \text { eff }}$ were measured and found to be $3.27 \pm 0.17 \times 10^{-8} \mathrm{~cm} / \mathrm{W}, 6.41 \pm 0.17 \times 10^{-8} \mathrm{~cm} / \mathrm{W}$ and $3.22 \pm 0.17 \times 10^{-8} \mathrm{~cm} / \mathrm{W}$ for $600 \mathrm{~nm}, 700 \mathrm{~nm}$ and $800 \mathrm{~nm}$ pumping wavelengths, respectively. To take into account the influence of nonlinear scattering, absorptiondependent photoacoustic measurements were carried out together with the optical Z-scan and the obtained data were compared. The origin of the effective absorptive non-linearity is discussed based on combined nonlinear absorption in NPs, nonlinear scattering, and bubble generation triggered by NPs-mediated light absorption. Potential applications include biomedical diagnostics and therapy (c) 2020 Optical Society of America
\end{abstract}

http://dx.doi.org/10.1364/OL.99.099999

Titanium nitride (TiN) nanoparticles (NPs) present an alternative plasmonic material to noble metal counterparts, with a red-shifted plasmonic extinction band (600-800 nm) compared to Au and Ag NPs (520-550 $\mathrm{nm}$ and $400-420 \mathrm{~nm}$, respectively) [1-3]. This feature makes them an attractive candidate for a variety of applications such as solar heat transducers [4], waveguides in the telecommunication range [5], functional arrays [6], electrochemical supercapacitors [7], etc. Biomedicine looks as one of main beneficiaries of such remarkable plasmonic absorption, falling in the first biological transparency window (630-900 nm) even for relatively small NP sizes. As an example, TiN NPs have been successfully used as photothermal agents for cancer therapy and in vivo imaging [8-10]. The mechanism enabling such biomedical applications arises from linear optical absorption near the plasmonic resonance, which provides contrast in bioimaging [11] and initiate a photothermal effect [12]. On the other hand, nonlinear optical (NLO) responses measured by optical Z-scan or other techniques can acquire important information on nonlinear absorption (NLA) and other characteristics, which can also help to unravel novel prospects for the development of imaging and therapy modalities. Such an expectation is confirmed by results of recent optical Z-scan studies reporting a strong NLO response using TiN NPs $[13,14]$.

Photoacoustic (PA) response of absorptive materials provides another attractive opportunity for NPs characterization, which arises from the transformation of absorbed photons into heat. Such a phenomenon forces microscopic expansions and subsequent contractions (after cooling), which in turn generate acoustic waves in the surrounding medium. There is a myriad of applications of photoacoustic techniques, from spectroscopy [15] to multiscale imaging and tomography [16]. When the photoacoustic process takes place in a liquid ambience, nanobubbles and microbubbles can also be generated [17,18]. Photoacoustic Z-scan (PAZ-scan) presents a modification of optical Z-scan, which detects the generation of photoacoustic waves in the NLO regime, while an optical pulse is nonlinearly absorbed by the sample and converted into sound waves [19]. The method has advantages over the optical Z-scan, since it relies solely on the material's absorption, can work with opaque samples and be used with a wide range of excitation wavelength, while scattering does not contribute to the recorded signals. An advanced implementation of this technique, termed OPAZ, combines optical Zscan and PAZ-scan to obtain a better understanding of the NLA in optical materials [20].

In this letter, for the first time we report a quantitative characterization of nonlinear photoacoustic response (NPR) of suspensions of laser-synthesized TiN NPs using OPAZ-scan. The lasersynthesized NPs were chosen as a new appealing object for biomedical applications, combining unique physico-chemical characteristics (spherical shape, controllable size under low size dispersion, absence of toxic impurities, high stability in aqueous or organic solvents, etc.) [21] with high safety in biological models in vitro and in vivo [22], which makes them advantageous compared to counterparts prepared by conventional methods, including chemical synthesis [1], epitaxy growth [6], electrochemical [7] and hydrothermal synthesis [8]. We measured effective nonlinear absorption coefficients $\beta$ PA,eff and discuss 
the role of nonlinear loss mechanisms, as a part of novel contributions of this work.

Solutions of TiN NPs were synthesized by methods of femtosecond (fs) laser ablation from a solid target in liquid ambience, which were earlier successfully applied for the fabrication of a variety of nanomaterials, including Au NPs [23,24] and Si NPs [25,26]. Briefly, a TiN target (GoodFellow, hot pressed, $99.5 \%$ purity) was placed vertically in a glass cuvette and immersed in acetone (Sigma Aldrich, laboratory reagent, $>99.5 \%$ purity), as schematically shown in Fig. 1(a). A laser beam from a Yb:KGW laser $(1030 \mathrm{~nm}, 270 \mathrm{fs}$ pulse length, $100 \mu \mathrm{J}$ pulse energy, $10 \mathrm{kHz}$ repetition rate, TETA 10 model, Avesta, Russia) was focused via a F-theta lens having $100 \mathrm{~mm}$ focal distance. The ablation time was $20 \mathrm{~min}$. Mass concentration of the obtained colloid was $50 \mu \mathrm{g} / \mathrm{mL}$, as determined by gravimetric method.
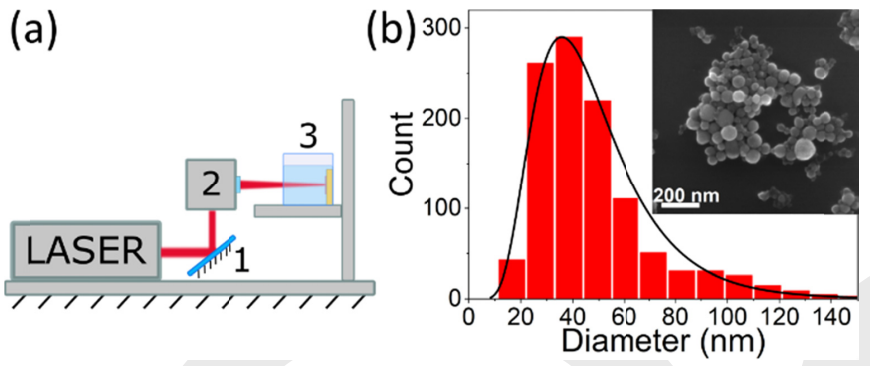

Fig. 1. (a) Schematic of laser synthesis. 1 -mirror, 2 -galvanometer scanner and F-theta lens, which make possible constant movement of the focal spot over the target, 3 - ablation chamber with a target. (b) An SEM image (inset) and size distribution of laser-synthesized TiN NPs

Structural properties and chemical composition were measured by a scanning transmission electron microscopy (STEM) system (MAIA 3, Tescan, Czech Republic) coupled with an EDS detector (X-act, Oxford Inst., UK). STEM confirmed the formation of spherical NPs (inset of Fig. 1b). Statistical analysis of STEM images revealed a log-normal size distribution with $40 \mathrm{~nm}$ mode size and $40 \mathrm{~nm}$ full-width-at-halfmaximum (Fig. 1b). EDS analysis revealed titanium and nitrogen as main elements in the composition of formed NPs, while signals corresponding to other elements $(\mathrm{O}, \mathrm{Si})$ originated from the substrate and titanium oxide coating of NPs surface (Fig. 2a).
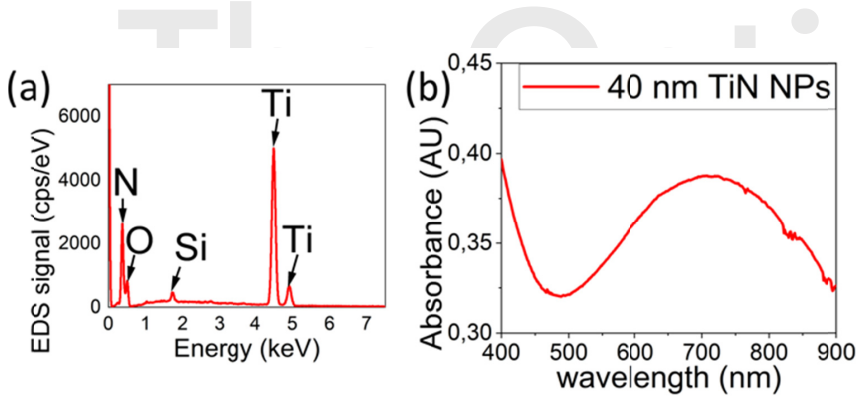

Fig. 2. EDS (a) and optical absorption (b) spectra of $40 \mathrm{~nm}$ TiN NPs.

Optical characterization of NPs by UV-Vis-NIR spectrophotometer (FluoroMax, Horiba) revealed a strong absorption in the ultraviolet for wavelengths below $400 \mathrm{~nm}$ due to interband transitions [13] and a broad extinction band around 600-900 $\mathrm{nm}$ due to the excitation of surface plasmon resonances (SPR) by TiN NPs (Fig. 2b) with mass extinction coefficient at plasmonic peak $18 \mathrm{~L} /\left(\mathrm{g}^{*} \mathrm{~cm}\right)$. This value is comparable to the extinction coefficient of $40 \mathrm{~nm} \mathrm{Au} \mathrm{NPs}\left(25 \mathrm{~L} /\left(\mathrm{g}^{*} \mathrm{~cm}\right)\right.$ [27]), although it is lower than that of $40 \mathrm{~nm} \mathrm{Ag} \mathrm{NPs}\left(159 \mathrm{~L} /\left(\mathrm{g}^{*} \mathrm{~cm}\right)\right.$
[28]) which is a champion in this field. More importantly, the mass extinction coefficient of TiN NPs is very high among materials having a plasmonic feature in near IR region: TiN NPs outperform Au nanorods of similar size (14 L/( $\left.\left.\mathrm{g}^{*} \mathrm{~cm}\right)[29]\right)$ and have slightly lower extinction than huge $150 \mathrm{~nm}$ Au based core-shell NPs (21 L/(g*cm) [30]).

The experimental setup for the OPAZ-scan is shown in Fig. 3. As the optical source, we used an optical parametric oscillator (OPO) which was tuned to three different wavelengths: $600 \mathrm{~nm}, 700 \mathrm{~nm}$ and 800 $\mathrm{nm}$, operating at $10 \mathrm{~Hz}$ and $5 \mathrm{~ns}$ pulses. The incident power was controlled by two Glan polarizers, followed by a telescope, used to optimize the beam diameter. The beam was split into two channels. The first one was sent to a reference photodetector (PD1) to control the input power, while the second one was focused onto the sample using a $125 \mathrm{~mm}$ focal length lens. In contrast to conventional optical Zscan, where the sample moves through the focus of the lens, in our case the lens was placed on a motorized translation stage, which was moved in small steps to enable scanning of the sample along the Z-axis. The TiN colloid was placed in a $2 \mathrm{~mm}$ quartz cuvette at 45 degrees to $\mathrm{Z}$ axis and was mounted in a cell containing distilled water for ultrasound coupling. The reflected PA signal was detected using a 10 $\mathrm{MHz}$ water immersion transducer (Olympus V312-N-SU) in front of the surface of the sample for a better coupling. The transmitted beam was collected by a photodetector (PD2) for the measurement of the open aperture Z-scan. The beam diameter at the focus $(\mathrm{z}=0)$ was $\mathrm{w}_{0}=$ $30 \mu \mathrm{m}$.

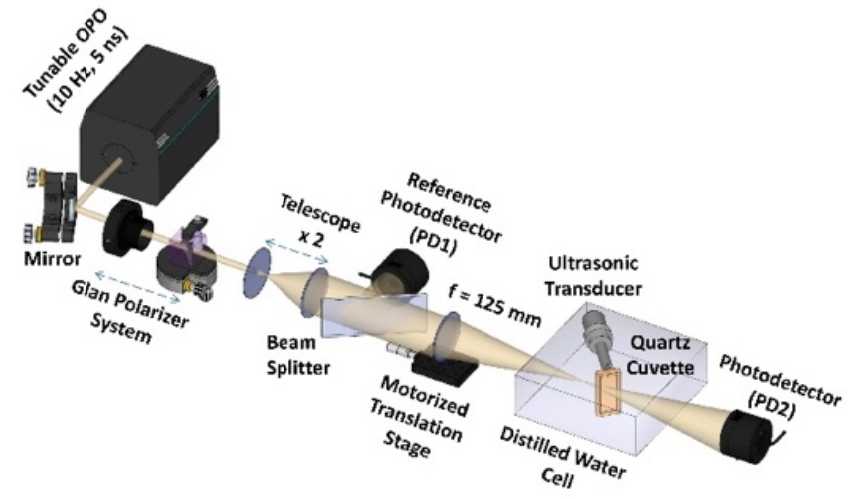

Fig. 3. Experimental setup to simultaneously measure open Z-scan and photoacoustic Z-scan (OPAZ). See text for further details.

The results of OPAZ-scan for the TiN colloid are shown in Fig 4. The left column (Fig. 4a, 4c, 4e) shows the photoacoustic signal for three different fluences, at three different wavelengths, whereas the simultaneously measured open aperture optical Z-scan is shown in the right column, (Fig. 4b, 4d, 4f). The data were obtained at fluences ranging from $0.71 \mathrm{~J} / \mathrm{cm}^{2}$ up to $1.32 \mathrm{~J} / \mathrm{cm}^{2}$, and the signal-to-noise ratio was much better in the case of the PAZ-scan. The measurements were reproducible, and Fig. 4 shows a representative example. The optical Zscan signal obtained with open aperture directly implies an absorptive (rather than saturated absorption) process. The photoacoustic signal is naturally inverted, since it is solely due to absorption while the open aperture Z-scan signal can be due to both absorption and scattering.

To understand our results, it is important to verify the excitation energy compared to the TiN bandgap, which can be calculated from the well-known Tauc's equation [31], reproduced in eq. 1:

$$
(\alpha h v)^{1 / r}=A\left(h v-E_{g}\right)
$$

where $\alpha$ is the linear absorption coefficient, A is the optical constant and $r=\mathbf{1} / 2$ for direct allowed transitions. Using linear absorption data 
from Fig. 2(b), the gap energy was calculated to be about $3.7 \mathrm{eV}$, similar to results for TiN films [13]. To induce a transition between $\mathrm{d}$ bands and the conduction band of TiN, the photon energy must be larger than the band gap energy $\left(\mathrm{Eg}_{\mathrm{g}}\right)$. In this work, the excitation energies $(2.06 \mathrm{eV}$, $1.77 \mathrm{eV}$, and $1.5 \mathrm{eV}$ ) were lower than the Eg. Therefore, electrons in the conduction band and two-photon-induced d electrons contributed to the nonlinear response the plasmon band, just like in [13,32]. We also expected a contribution of plasmon resonances at high intensities as the excitation wavelength was in the resonant region of the sample. From the obtained results, we can anticipate that the plasmonic effect appears at the center of the plasmonic band, around $700 \mathrm{~nm}$, where the NPR was clearly higher than at the other two wavelengths (see table I). This effect did not appear in the optical domain, probably because of the small scattering effect leading to the differences in the values of NLA coefficient obtained by the optical and photoacoustic Zscan. The role of a plasmonic enhancement in TiN is therefore clear in the NLA process, similar to that in Au NPs. However, we believe that the dominant effect for the NLA in our sample is the TPA for all intensities.

\section{$600 \mathrm{~nm}$}
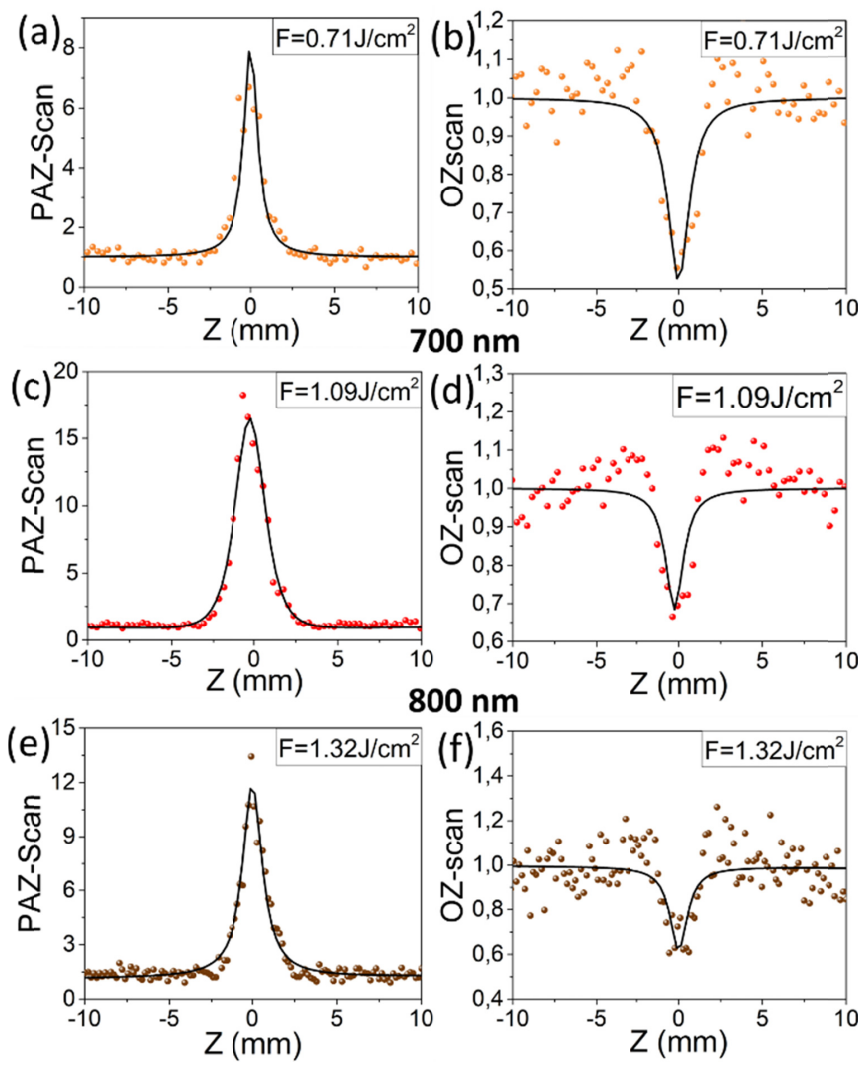

Fig. 4. Experimental results (dots) and theoretical fits (lines) of the NLR from TiN NP with the OPAZ setup. Left column (a-c): photoacoustic Z-scan measurements; right column (d-f): optical Z-scan with open aperture, simultaneously measured.

To calculate the nonlinear coefficients arising from the nonlinear absorptive response, we employed the open aperture optical Z-scan curve using the eq. (2) [33]:

$$
T_{\text {OZscan }}=\sum_{m=0}^{\infty} \frac{1}{(m+1)^{3 / 2}}\left(\frac{-\beta I_{0} L_{e f f}}{\left[1+\left(z / z_{0}\right)^{2}\right]}\right)^{m}
$$

The normalized transmission is proportional to the NLA coefficient, $\beta$. $\mathrm{I}_{0}$ is the intensity at focus, $L_{e f f}=\frac{1-e^{-\alpha_{0} L}}{\alpha_{0}}$ is the effective thickness of the sample with a linear coefficient $\alpha_{0}$, L is the sample thickness, and $\mathrm{z}_{0}$ is the Rayleigh length of the beam. In eq. (2), the effect of nonlinear scattering was neglected.

On the other hand, in the PAZ-scan, the acoustic signal amplitude $P$ is proportional to the absorption coefficient $\mu_{a}$, i.e.,

$$
P=\Gamma \mu_{a} I,
$$

where $I$ is the incident light intensity, and $\Gamma$ is the Grüneisen coefficient which depends on the material properties and the laser beam characteristics. The PA signal is independent of the scattering process; thus, we can expect that it describes pure light absorptive nature of the process. In this case, the photoacoustic signal is produced by the complex interaction between intense incident light and TiN NPs. This interaction can include the nonlinear absorption by NPs, but also the generation of bubbles around NPs due to their high photoheating, therefore we replaced $\mu_{a}$ to include the effective nonlinear absorption coefficient. Then,

$$
\boldsymbol{\mu}_{\boldsymbol{a}}=\left(\boldsymbol{\alpha}_{0}+\boldsymbol{\beta} \boldsymbol{I}\right)
$$

The normalized photoacoustic signal for PAZ-scan is given by $[17,18]$, with the PA signal normalized by the far-field component:

$$
P_{\text {Normalized }}=1+\frac{\beta}{\alpha_{0}} \frac{I_{0}}{\left[1+\left(z / z_{0}\right)^{2}\right]}
$$

In a NPs suspension, as in our case, the absorptive effects can be influenced by losses arising from nonlinear scattering [34], or due to the formation of microbubbles [16-18], although the effect of microbubbles is often neglected in such measurements. Since the optical Z-scan does not measure the influence of either of those two mechanisms, while the PAZ-scan is not affected by scattering, we cannot directly differentiate the contribution of microbubbles from the one of NLA of NPs.

In the first approximation, the values for an effective NLA coefficient can be obtained from eqs. $(1-5)$. These values are given in table I. We

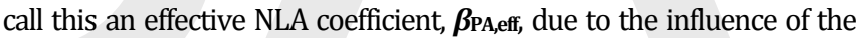
above-mentioned mechanisms leading to the NPR, as detected by photoacoustics. Table I also shows results of the optical Z-scan, which can be compared to other literature values. Notice that, although one should be careful when comparing the data, since TiN are in different formats (NPs or thin film) and different spectro-temporal excitation regimes, some qualitative comparison can be made. From table I, the NLA coefficient obtained from optical Z-scan results for the TiN NPs suspension is within the same order of magnitude to those obtained in powders at similar pulse duration, but different excitation wavelength [13]. In the case of TiN thin films in the femtosecond regime [14], the optical NLA was one order of magnitude higher than our values obtained in nanosecond regime. One reason could be the absence of losses due to scattering and bubble formation, since it is not a colloid. Another important aspect is that the obtained values from the optical measurements are also not far from those obtained from the photoacoustic Z-scan. As mentioned before, the PAZ-scan is not sensitive to scattering effect. Therefore, one can conclude that nonlinear scattering may slightly affects the result and it is wavelength dependent. At $600 \mathrm{~nm}$, $\beta_{\mathrm{PA} \text {,eff }} \sim 1.36 \beta_{\text {OZ-SCAN, }}$ at $700 \mathrm{~nm}$ it grows to $\beta_{\mathrm{PA} \text {,eff }}$ $\sim 6.16 \beta$ OZ-SCAN, and at $800 \mathrm{~nm}$ it is $\beta$ PA,eff $\sim 2.62 \beta_{\text {OZ-SCAN }}$. The increase of $\beta_{\text {PA,eff }}$ to $\beta_{\text {OZ-SCAN ratio near the plasmonic resonance indicates significant }}$ increase of relative portion of absorption in total extinction, or, in other words, high extinction in the plasmonic peak is due to increase of absorption rather than increase of scattering. This fact is beneficial for projected applications of TiN NPs colloids. The higher nonlinear coefficient at the plasmonic band central resonance confirms the role 
of plasmonic enhancement. On the other hand, one important issue is related to the fact that in the photoacoustic measurements, the photons absorbed by TiN NPs are transformed into heat, which is very likely to create microbubbles. In order to clarify the role of such microbubbles, time resolved studies should be performed, as in $[16,17]$, where microbubbles in gold colloids were studied. Therefore, the NLA values obtained in our work could be affected by this phenomenon, and further experiments are required to verify this possibility.

Table I Calculated values of the NLA coefficient for TiN NPs in this work and comparison with literature values

\begin{tabular}{|c|c|c|c|c|c|c|}
\hline $\begin{array}{c}\text { Sam } \\
\text { ple }\end{array}$ & $\begin{array}{c}\text { Preparatio } \\
\text { n method/ } \\
\text { solvent }\end{array}$ & $\begin{array}{c}\lambda, \\
\mathrm{nm}\end{array}$ & $\begin{array}{c}\mathrm{NP} \\
\text { size, } \\
\mathrm{nm}\end{array}$ & $\begin{array}{l}\beta_{\mathrm{PA}, \mathrm{eff}} \\
\times 10^{-8}, \\
\mathrm{~cm} / \mathrm{W}\end{array}$ & $\begin{array}{c}\text { BOZ- } \\
\text { SCAN } \\
\times 10^{-8} \\
\mathrm{~cm} / \mathrm{W}\end{array}$ & $\begin{array}{c}\text { Ref } \\
\left(\tau_{\text {exc }}\right)\end{array}$ \\
\hline TiN & $\begin{array}{c}\text { Laser } \\
\text { ablation/ } \\
\text { acetone }\end{array}$ & $\begin{array}{l}600 \\
700 \\
800\end{array}$ & $\begin{array}{l}40 \\
40 \\
40\end{array}$ & $\begin{array}{c}3.27 \\
\pm 0.17 \\
6.41 \\
\pm 0.32 \\
3.22 \\
\pm 0.16 \\
\end{array}$ & $\begin{array}{l}2.40 \\
1.04 \\
1.23\end{array}$ & $\begin{array}{l}\text { This } \\
\text { work } \\
\text { (5 ns) }\end{array}$ \\
\hline TiN & $\begin{array}{l}\text { Commerci } \\
\text { al powder }\end{array}$ & 532 & 55 & --- & 8.31 & $\begin{array}{l}{[13]^{\mathrm{A}}} \\
(7 \mathrm{~ns})\end{array}$ \\
\hline TiN & Thin film & 780 & --- & --- & -68 & $\begin{array}{c}{[14]^{\mathrm{B}}} \\
(150 \\
\mathrm{fs}, \\
1 \mathrm{kHz}) \\
\end{array}$ \\
\hline TiN & Thin film & $\begin{array}{c}155 \\
0\end{array}$ & --- & --- & -66 & $\begin{array}{c}{[14]} \\
(150 \\
\mathrm{fs}, \\
1 \mathrm{kHz}) \\
\end{array}$ \\
\hline
\end{tabular}

AExcitation intensity one order of magnitude higher than in the present work. B52nm thick thin film.

In conclusion, effective values of optically induced nonlinear photoacoustics response of suspensions of $40 \mathrm{~nm}$ TiN NPs were reported for the first time using a combined technique for simultaneous optical and photoacoustic detection. Using the OPAZscan method, it was shown that nonlinear scattering can affect the results, as a loss mechanism influencing the nonlinear absorption. Furthermore, bubble formation due to heating cannot be ruled out and further experiments are required to clarify this issue. Such bubbling effects are important for NPs mediated laser ablation of biological tissues and can be used for potential theranostics applications [16]. The plasmonic enhancement in the TiN NPs can also be exploited for biomedical applications such as photothermal therapy [9,10,21], which can be extended by serial PA tomography [35], as demonstrated with gold NP.

Funding. This work was funded by INCT of Photonics Program supported by CNPq, CAPES and FACEPE (Grant 465.763/2014-6) for optical characterization and the Russian Science Foundation (Project 19-72-30012) for fabrication and characterization of TiN NPs.

Acknowledgements. A.D. thanks the PNPD Program, CAPES/UFPE, for a post-doctoral fellowship. ASLG thanks CNPq for grant PQ-1A 307259/2015-3.

Disclosures. The authors declare no conflicts of interest.

\section{References}

1. S. Kaskel, K. Schlichte, G. Chaplais, and M. Khanna, J. Mater. Chem. 13, 1496 (2003).

2. U. Guler, S. Suslov, A. V. Kildishev, A. Boltasseva, and V. M. Shalaev, Nanophotonics 4, 269 (2015).

3. A. Lalisse, G. Tessier, J. Plain, and G. Baffou, Sci. Rep. 6, 38647 (2016).

4. S. Ishii, R. P. Sugavaneshwar, and T. Nagao, J. Phys. Chem. C 120, 2343 (2016).

5. V. I. Zakomirnyi, I. L. Rasskazov, V. S. Gerasimov, A. E. Ershov, S. P. Polyutov, S. V. Karpov, and H. Ågren, Photonics Nanostructures Fundam. Appl. 30, 50 (2018).

6. S. Murai, K. Fujita, Y. Daido, R. Yasuhara, R. Kamakura, and K. Tanaka, Opt. Express 24, 1143 (2016).

7. S. A. Ansari, N. A. Khan, Z. Hasan, A. A. Shaikh, F. K. Ferdousi, H. R. Barai, N. S. Lopa, and M. M. Rahman, Sustain. Energy Fuels 4, 2480 (2020).

8. W. Jiang, Q. Fu, H. Wei, and A. Yao, J. Mater. Sci. 54, 5743 (2019).

9. W. He, K. Ai, C. Jiang, Y. Li, X. Song, and L. Lu, Biomaterials 132, 37 (2017).

10. C. Wang, C. Dai, Z. Hu, H. Li, L. Yu, H. Lin, J. Bai, and Y. Chen, Nanoscale Horizons 4, 415 (2019).

11. A. Kopwitthaya, K.-T. Yong, R. Hu, I. Roy, H. Ding, L. A. Vathy, E. J. Bergey, and P. N. Prasad, Nanotechnology 21, 315101 (2010).

12. D. Jaque, L. Martínez Maestro, B. del Rosal, P. Haro-Gonzalez, A. Benayas, J. L. Plaza, E. Martín Rodríguez, and J. García Solé, Nanoscale 6, 9494 (2014).

13. S. Divya, V. P. N. Nampoori, P. Radhakrishnan, and A. Mujeeb, Laser Phys. Lett. 11, 085401 (2014).

14. N. Kinsey, A. A. Syed, D. Courtwright, C. DeVault, C. E. Bonner, V. I. Gavrilenko, V. M. Shalaev, D. J. Hagan, E. W. Van Stryland, and A. Boltasseva, Opt. Mater. Express 5, 2395 (2015).

15. C. Haisch, Meas. Sci. Technol. 23, 012001 (2012).

16. S. Wang, L. Fu, J. Xin, S. Wang, C. Yao, Z. Zhang, and J. Wang, J. Biomed. Opt. 23, 1 (2018).

17. D. Lapotko, Opt. Express 17, 2538 (2009).

18. S. Kudryashov, A. Samokhvalov, A. Nastulyavichus, I. Saraeva, V. Mikhailovskii, A. Ionin, and V. Veiko, Materials. 12, 562 (2019).

19. C. Yelleswarapu and S.-R. Kothapalli, Opt. Express 18, 9020 (2010).

20. P. Chantharasupawong, R. Philip, and J. Thomas, Appl. Phys. Lett. 102, 041116 (2013).

21. A. A. Popov, G. Tselikov, N. Dumas, C. Berard, K. Metwally, N. Jones, A. Al-Kattan, B. Larrat, D. Braguer, S. Mensah, A. Da Silva, M.A. Estève, and A. V. Kabashin, Sci. Rep. 9, 1194 (2019).

22. I. V. Zelepukin, A. A. Popov, V. O. Shipunova, G. V. Tikhonowski, E. A. Mirkasymov, E. A. Popova-Kuznetsova, S. M. Klimentov, A. V. Kabashin, and S. M. Deyev, Mater. Sci. Eng. C, in press, (2020).

23. S. Hebié, Y. Holade, K. Maximova, M. Sentis, P. Delaporte, K. B. Kokoh, T. W. Napporn, A. V. Kabashin, ACS Catal. 5, 6489 (2015).

24. A. Bailly, F. Correard, A. Popov, G. Tselikov, F. Chaspoul, R. Appay, A. Al-kattan, A. V Kabashin, and D. Braguer, Sci. Rep. 1 (2019).

25. T. Baati, A. Al-Kattan, M.-A. Esteve, L. Njim, Y. Ryabchikov, F. Chaspoul, M. Hammami, M. Sentis, A. V. Kabashin, and D. Braguer, Sci. Rep. 6, 25400 (2016).

26. A. Al-Kattan, Y. V Ryabchikov, T. Baati, V. Chirvony, J. F. SánchezRoyo, M. Sentis, D. Braguer, V. Y. Timoshenko, M.-A. Estève, and A. V Kabashin, J. Mater. Chem. B 4, 7852 (2016).

27. W. Haiss, N. T. K. Thanh, J. Aveyard, and D. G. Fernig, Anal. Chem. 79, 4215 (2007).

28. D. Paramelle, A. Sadovoy, S. Gorelik, P. Free, J. Hobley, and D. G. Fernig, Analyst 139, 4855 (2014).

29. J. T. Robinson, K. Welsher, S. M. Tabakman, S. P. Sherlock, H. Wang, R. Luong, and H. Dai, Nano Res. 3, 779 (2010).

30. S. C. Gad, K. L. Sharp, C. Montgomery, J. D. Payne, and G. P. Goodrich, Int. J. Toxicol. 31, 584 (2012). 
31. J. Tauc, R. Grigorovici, and A. Vancu, Phys. Status Solidi 15, 627 (1966).

32. Y. Wan and L. Deng, Appl. Sci. 10, 199 (2020).

33. C. B. de Araújo, A. S. L. Gomes, and G. Boudebs, Reports Prog. Phys. 79, 036401 (2016).

34. N. T. C. Oliveira, A. S. Reyna, E. H. L. Falcão, and C. B. de Araújo, J. Phys. Chem. C 123, acs. jpcc.9b01369 (2019).

35. S. Schrof, G. Pang, J. Buchmann, and J. Laufer, J. Imaging 4, 146 (2018).

\section{References (with titles)}

1. S. Kaskel, K. Schlichte, G. Chaplais, and M. Khanna, "Synthesis and characterisation of titanium nitride based nanoparticles," J. Mater. Chem. 13, 1496 (2003).

2. U. Guler, S. Suslov, A. V. Kildishev, A. Boltasseva, and V. M. Shalaev, "Colloidal Plasmonic Titanium Nitride Nanoparticles: Properties and Applications," Nanophotonics 4, 269-276 (2015).

3. A. Lalisse, G. Tessier, J. Plain, and G. Baffou, "Plasmonic efficiencies of nanoparticles made of metal nitrides (TiN, ZrN) compared with gold," Sci. Rep. 6, 38647 (2016).

4. S. Ishii, R. P. Sugavaneshwar, and T. Nagao, "Titanium Nitride Nanoparticles as Plasmonic Solar Heat Transducers," J. Phys. Chem. C 120, 2343-2348 (2016).

5. V. I. Zakomirnyi, I. L. Rasskazov, V. S. Gerasimov, A. E. Ershov, S. P. Polyutov, S. V. Karpov, and H. Ågren, "Titanium nitride nanoparticles as an alternative platform for plasmonic waveguides in the visible and telecommunication wavelength ranges," Photonics Nanostructures - Fundam. Appl. 30, 50-56 (2018).

6. S. Murai, K. Fujita, Y. Daido, R. Yasuhara, R. Kamakura, and K. Tanaka, "Plasmonic arrays of titanium nitride nanoparticles fabricated from epitaxial thin films," Opt. Express 24, 1143 (2016).

7. S. A. Ansari, N. A. Khan, Z. Hasan, A. A. Shaikh, F. K. Ferdousi, H. R. Barai, N. S. Lopa, and M. M. Rahman, "Electrochemical synthesis of titanium nitride nanoparticles onto titanium foil for electrochemical supercapacitors with ultrafast charge/discharge," Sustain. Energy Fuels 4, 2480-2490 (2020).

8. W. Jiang, Q. Fu, H. Wei, and A. Yao, "TiN nanoparticles: synthesis and application as near-infrared photothermal agents for cancer therapy," J. Mater. Sci. 54, 5743-5756 (2019).

9. W. He, K. Ai, C. Jiang, Y. Li, X. Song, and L. Lu, "Plasmonic titanium nitride nanoparticles for in vivo photoacoustic tomography imaging and photothermal cancer therapy," Biomaterials 132, 37-47 (2017).

10. C. Wang, C. Dai, Z. Hu, H. Li, L. Yu, H. Lin, J. Bai, and Y. Chen, "Photonic cancer nanomedicine using the near infrared-II biowindow enabled by biocompatible titanium nitride nanoplatforms," Nanoscale Horizons 4, 415-425 (2019).

11. A. Kopwitthaya, K.-T. Yong, R. Hu, I. Roy, H. Ding, L. A. Vathy, E. J. Bergey, and P. N. Prasad, "Biocompatible PEGylated gold nanorods as colored contrast agents for targeted in vivo cancer applications," Nanotechnology 21, 315101 (2010).

12. D. Jaque, L. Martínez Maestro, B. del Rosal, P. Haro-Gonzalez, A. Benayas, J. L. Plaza, E. Martín Rodríguez, and J. García Solé, "Nanoparticles for photothermal therapies," Nanoscale 6, 94949530 (2014).

13. S. Divya, V. P. N. Nampoori, P. Radhakrishnan, and A. Mujeeb, "Origin of optical non-linear response in TiN owing to excitation dynamics of surface plasmon resonance electronic oscillations," Laser Phys. Lett. 11, 085401 (2014).

14. N. Kinsey, A. A. Syed, D. Courtwright, C. DeVault, C. E. Bonner, V. I. Gavrilenko, V. M. Shalaev, D. J. Hagan, E. W. Van Stryland, and A. Boltasseva, "Effective third-order nonlinearities in metallic refractory titanium nitride thin films," Opt. Mater. Express 5, 2395 (2015).

15. C. Haisch, "Photoacoustic spectroscopy for analytical measurements," Meas. Sci. Technol. 23, 012001 (2012).

16. S. Wang, L. Fu, J. Xin, S. Wang, C. Yao, Z. Zhang, and J. Wang, "Photoacoustic response induced by nanoparticle-mediated photothermal bubbles beyond the thermal expansion for potential theranostics," J. Biomed. Opt. 23, 1 (2018).

17. D. Lapotko, "Optical excitation and detection of vapor bubbles around plasmonic nanoparticles," Opt. Express 17, 2538 (2009).

18. S. Kudryashov, A. Samokhvalov, A. Nastulyavichus, I. Saraeva, V. Mikhailovskii, A. lonin, and V. Veiko, "Nanosecond-Laser Generation of Nanoparticles in Liquids: From Ablation through Bubble Dynamics to Nanoparticle Yield," Materials (Basel). 12, 562 (2019).

19. C. S. Yelleswarapu and S.-R. Kothapalli, "Nonlinear photoacoustics for measuring the nonlinear optical absorption coefficient," Opt. Express 18, 9020 (2010)

20. P. Chantharasupawong, R. Philip, and J. Thomas, "Simultaneous optical and photoacoustic measurement of nonlinear absorption," Appl. Phys. Lett. 102, 041116 (2013).

21. A. A. Popov, G. Tselikov, N. Dumas, C. Berard, K. Metwally, N. Jones, A. Al-Kattan, B. Larrat, D. Braguer, S. Mensah, A. Da Silva, M.A. Estève, and A. V. Kabashin, "Laser- synthesized TiN nanoparticles as promising plasmonic alternative for biomedical applications," Sci. Rep. 9, 1194 (2019).

22. I. V. Zelepukin, A. A. Popov, V. O. Shipunova, G. V. Tikhonowski, E. A. Mirkasymov, E. A. Popova-Kuznetsova, S. M. Klimentov, A. V. Kabashin, and S. M. Deyev, "Laser-synthesized TiN nanoparticles for biomedical applications: evaluation of safety, biodistribution and pharmacokinetics," Mater. Sci. Eng. C, in press, (2020).

23. S. Hebié, Y. Holade, K. Maximova, M. Sentis, P. Delaporte, K. B. Kokoh, T. W. Napporn, and A. V. Kabashin, "Advanced electrocatalysts on the basis of bare Au nanomaterials for biofuel cell applications," ACS Catal. 5, 6489-6496 (2015).

24. A. Bailly, F. Correard, A. Popov, G. Tselikov, F. Chaspoul, R. Appay, A. Al-kattan, A. V Kabashin, and D. Braguer, "In vivo evaluation of safety, biodistribution and pharmacokinetics of laser- synthesized gold nanoparticles," Sci. Rep. 1-12 (2019).

25. T. Baati, A. Al-Kattan, M.-A. Esteve, L. Njim, Y. Ryabchikov, F. Chaspoul, M. Hammami, M. Sentis, A. V. Kabashin, and D. Braguer, "Ultrapure laser-synthesized Si-based nanomaterials for biomedical applications: in vivo assessment of safety and biodistribution," Sci. Rep. 6, 25400 (2016).

26. A. Al-Kattan, Y. V Ryabchikov, T. Baati, V. Chirvony, J. F. SánchezRoyo, M. Sentis, D. Braguer, V. Y. Timoshenko, M.-A. Estève, and A. V Kabashin, "Ultrapure laser-synthesized Si nanoparticles with variable oxidation states for biomedical applications," J. Mater. Chem. B 4, 7852-7858 (2016).

27. W. Haiss, N. T. K. Thanh, J. Aveyard, and D. G. Fernig, "Determination of Size and Concentration of Gold Nanoparticles from UV-Vis Spectra," Anal. Chem. 79, 4215-4221 (2007).

28. D. Paramelle, A. Sadovoy, S. Gorelik, P. Free, J. Hobley, and D. G. Fernig, "A rapid method to estimate the concentration of citrate capped silver nanoparticles from UV-visible light spectra," Analyst 139, 4855 (2014).

29. J. T. Robinson, K. Welsher, S. M. Tabakman, S. P. Sherlock, H. Wang, R. Luong, and H. Dai, "High performance in vivo near-IR (>1 $\mu \mathrm{m}$ ) imaging and photothermal cancer therapy with carbon nanotubes," Nano Res. 3, 779-793 (2010).

30. S. C. Gad, K. L. Sharp, C. Montgomery, J. D. Payne, and G. P. Goodrich, "Evaluation of the Toxicity of Intravenous Delivery of Auroshell Particles (Gold-Silica Nanoshells)," Int. J. Toxicol. 31, 584594 (2012). 
31. J. Tauc, R. Grigorovici, and A. Vancu, "Optical Properties and Electronic Structure of Amorphous Germanium," Phys. status solidi 15, 627-637 (1966).

32. Y. Wan and L. Deng, "Pump-Controlled Plasmonic Random Lasers from Dye-Doped Nematic Liquid Crystals with TiN Nanoparticles in Non-Oriented Cells," Appl. Sci. 10, 199 (2020).

33. C. B. de Araújo, A. S. L. Gomes, and G. Boudebs, "Techniques for nonlinear optical characterization of materials: a review," Reports Prog. Phys. 79, 036401 (2016).

34. N. T. C. Oliveira, A. S. Reyna, E. H. L. Falcão, and C. B. de Araújo, "Light Scattering, Absorption, and Refraction due to High-Order Optical Nonlinearities in Colloidal Gold Nanorods," J. Phys. Chem. C 123, acs.jpcc.9b01369 (2019).

35. S. Schrof, G. Pang, J. Buchmann, and J. Laufer, "Exploiting Nonlinear Photoacoustic Signal Generation in Gold Nanospheres for Selective Detection in Serial 3D PA Tomography," J. Imaging 4, 146 (2018).
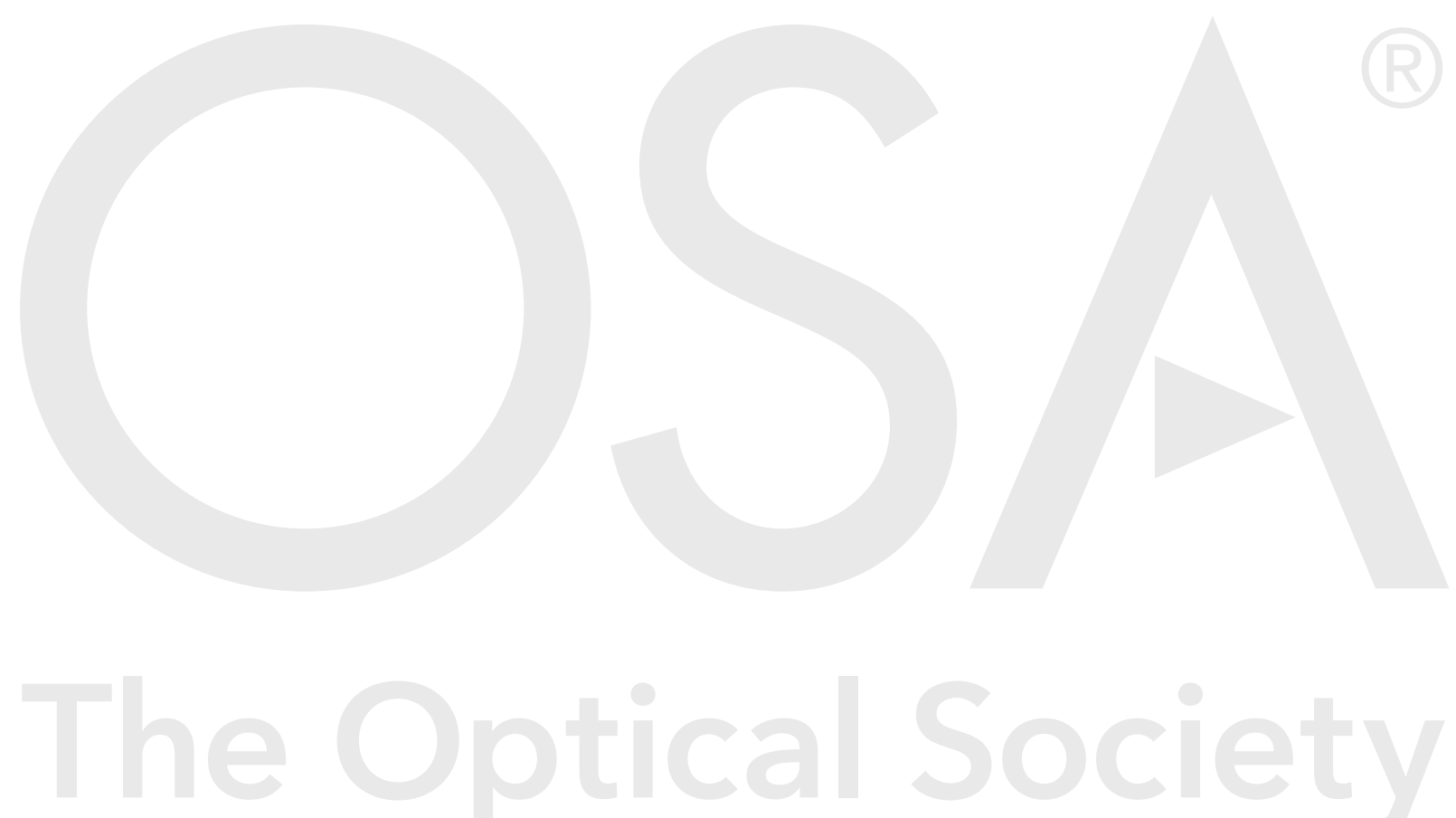